\title{
Commentary: EEG beta suppression and low gamma modulation are different elements of human upright walking
}

\author{
Carlos Trenado * \\ Institute of Clinical Neuroscience, Heinrich Heine University Düsseldorf, Düsseldorf, Germany
}

Keywords: gait, phase transition, modulation, index, analysis

\section{A commentary on}

EEG beta suppression and low gamma modulation are different elements of human upright walking

by Seeber, M., Scherer, R., Wagner, J., Solis-Escalante, T., and Müller-Putz, G. R. (2014). Front. Hum. Neurosci. 8:485. doi: 10.3389/fnhum.2014.00485

\section{OPEN ACCESS}

Edited by:

Pierfilippo De Sanctis,

Albert Einstein College of Medicine

USA

Reviewed by:

Adam C. Snyder,

University of Pittsburgh, USA

*Correspondence:

Carlos Trenado,

carlos.trenado@

med.uni-duesseldorf.de;

carlos.trenadoc@gmail.com

Received: 22 April 2015

Accepted: 15 June 2015

Published: 26 June 2015

Citation:

Trenado C (2015) Commentary: EEG beta suppression and low gamma modulation are different elements of human upright walking.

Front. Hum. Neurosci. 9:380. doi: 10.3389/fnhum.2015.00380
Recently Seeber and colleagues proposed a gait-phase modulation (GPM) signal to analyze the modulation of EEG oscillatory amplitudes relative to gait cycle. In particular, GPM was defined as a modified version of the previously introduced phase-amplitude coupling index (Canolty et al., 2006) under the assumption of a two-period pattern for studying upright walking:

$$
G P M=\frac{2}{\sqrt{2} \sigma_{A}(f)} \sum_{n=0}^{N-1} A(n, f) e^{-2 \pi i \frac{2 n}{N}} .
$$

As it is apparent from the mathematical definition and only after application of the absolute value on the obtained complex value for each frequency $f$, GPM enables quantification of phaselocking modulated oscillations from cortical regions in relation to periodic cycles in the frequency domain. Consequently, it potentially facilitates the determination of brain networks implicated in periodic body movements as in the case of upright walking by focusing on synchronization values corresponding to frequency bands of interest.

It is worth mentioning that quantification of brain synchronization through phase-locking dates back to the seminal works by Tass et al. (1998) and Lachaux et al. (1999) who introduced a realvalued bounded index (phase-locking value $P L V$ ) reflecting phase inter-trial variability between brain signals in the time-domain. By observing PLV patterns in relation to behavior, these authors were able to emphasize the role of phase synchronization as a putative mechanism for long-range neural integration in cognitive tasks which together with short-range phase synchrony commonly associated to perceptual binding represents an important principle for brain communication. Different studies highlighting the use of phase-locking in brain signals include: neural network connectivity during resting states in humans by magnetoencephalography (Schmidt et al., 2014), assessment of neural correlates of auditory selective attention reflected in electroencephalographic evoked potentials (Trenado et al., 2009), electroencephalographic correlates of listening effort (Strauss et al., 2010), electroencephalographic epilepsy studies (Mormann et al., 2000), studies dealing with brain computer interfaces (Brunner et al., 2006) and studies dealing with interpersonal body and neural synchronization as a marker of implicit social interaction (Yun et al., 2012). 
In spite of the usefulness and relevance of the GPM signal as previously stated, its definition does not meet the requirements of a phase-locking index as in the case of $P L V$, namely it should provide a real-value as an output and incorporate a scale factor to obtain bounded values in the interval $[0,1]$ without the need for pre-normalization. To address these issues while providing a more satisfactory definition of gait phase modulation index analogous to $P L V$, the following definition is suggested:

$$
G P M_{\sigma}=\frac{2}{N \sqrt{2} \sigma_{A}(f)}\left|\sum_{n=0}^{N-1} A(n, f) e^{-2 \pi i \frac{2 n}{N}}\right|
$$

where $N$ is the total number of samples per gait cycle, $A$ denotes the time-frequency magnitude at a certain frequency $f$ and sample point $n$, and $\sigma_{A}(f)$ denotes its standard deviation. In particular, the normalization factor of $G P M_{\sigma}$ aims to capture the proportion of power variation that is coherent with the carrier signal while in the case of $P L V$ such a normalization factor, e.g. the number of samples, is merely for scaling.

Focusing on the aim of $G P M_{\sigma}$, an alternative and perhaps more natural way to quantify gait-phase modulation could be given by estimating the correlation between the time-frequency magnitude modulation $A$ and the gait cycle, for instance by calculating the magnitude of the Pearson's correlation coefficient between the complex-valued analytic form of the time-frequency

\section{References}

Brunner, C., Scherer, R., Graimann, B., Supp, G., and Pfurtscheller, G. (2006). Online control of a brain computer interface using phase synchronization. IEEE Trans. Biomed. Eng. 53, 2501-2506. doi: 10.1109/TBME.2006. 881775

Canolty, R. T., Edwards, E., Dalal, S. S., Soltani, M., Nagarajan, S. S., Kirsch, H. E.,et al. (2006). High gamma power is phase-locked to theta oscillations in human neocortex. Science 313, 1626-1628. doi: 10.1126/science. 1128115

Lachaux, J.-P., Rodriguez, E., Martinerie, J., and Varela, F. J. (1999). Measuring the phase synchrony in brain signals. Hum. Brain Mapp. 8, 194-208. doi: 10.1002/(SICI)1097-0193(1999)8:4<194::AID-HBM4>3.0.CO;2-C

Mormann, F., Lehnertz, K., David, P. E., and Elger, C. (2000). Mean phase coherence as a measure for phase synchronization and its application to the EEG of epilepsy patients. Phys. D 144, 358-369. doi: 10.1016/S01672789(00)00087-7

Schmidt, B. T., Ghuman, A. S., and Huppert, T. J. (2014). Whole brain functional connectivity using phase locking measures of resting state magnetoencephalography. Front. Neurosci. 8:141. doi: 10.3389/fnins.2014.00141

Seeber, M., Scherer, R., Wagner, J., Solis-Escalante, T., and Müller-Putz, G. R. (2015). High and low gamma EEG oscillations in central sensorimotor areas are conversely modulated during the human gait cycle. Neuroimage 112, 318-326. doi: 10.1016/j.neuroimage.2015.03.045 magnitude $A$ and the complex-valued representation of the gait cycle.

In contrast to $P L V, G P M_{\sigma}$ addresses possible phase interactions between a brain signal and a sinusoidal signal representing a periodic motor movement. One of the main advantages of using phase analysis is the fact that it does not require any statistical assumption about the signals while being more robust against noise. An additional capability of this measure is the possibility to evaluate existing phase lags in the motor cycle which are hidden or overlooked by common correlation coefficients and coherence analysis as emphasized by Seeber et al. (2015).

Based on the previous, it is expected that the usefulness of the gait phase modulation index will be made apparent by future studies targeting extraction of neurophysiological correlates of human gait not only in the case of healthy individuals but importantly in patients with motor disorders.

\section{Acknowledgments}

Thanks to Lena Storzer for bringing to my attention the definition of the gait phase modulation index. CT is supported by ERA-NET NEURON/German Federal Ministry of Education and Research (BMBF) [TYMON 01EW141]. The author gratefully acknowledges the contribution of the reviewer on key aspects of the index.

Strauss, D. J., Corona-Strauss, F. I., Trenado, C., Bernarding, C., Reith, W., Latzel, M., et al. (2010). Electrophysiological correlates of listening effort: neurodynamical modeling and measurement. Cogn. Neurodyn. 4, 119-131. doi: 10.1007/s11571-010-9111-3

Tass, P., Rosenblum, M. G., Weule, J., Kurths, J., Pikovsky, A., Volkmann, J., et al. (1998). Detection of n:m Phase Locking from Noisy Data: Application to Magnetoencephalography. Phys. Rev. Lett. 81, 3291-3294. doi: 10.1103/PhysRevLett.81.3291

Trenado, C., Haab, L., and Strauss, D. J. (2009). Corticothalamic feedback dynamics for neural correlates of auditory selective attention. IEEE Trans. Neural Syst. Rehabil. Eng. 17, 46-52. doi: 10.1109/TNSRE.2008.2010469

Yun, K., Watanabe, K., and Shimojo, S. (2012). Interpersonal body and neural synchronization as a marker of implicit social interaction. Sci. Rep. 2:959. doi: $10.1038 /$ srep00959

Conflict of Interest Statement: The author declares that the research was conducted in the absence of any commercial or financial relationships that could be construed as a potential conflict of interest.

Copyright (C) 2015 Trenado. This is an open-access article distributed under the terms of the Creative Commons Attribution License (CC BY). The use, distribution or reproduction in other forums is permitted, provided the original author(s) or licensor are credited and that the original publication in this journal is cited, in accordance with accepted academic practice. No use, distribution or reproduction is permitted which does not comply with these terms. 